\title{
Adjuvant vaginal interventional radiotherapy in early-stage non-endometrioid carcinoma of corpus uteri: a systematic review
}

\begin{abstract}
Francesca De Felice, MD, PhD', Valentina Lancellotta, MD², Lisa Vicenzi, MD, PhD³, Sara Costantini, MD³, Alfredo Antonacci, MD ${ }^{4}$, Valentina Cerboneschi, MD5 , Daniela di Cristino, MD6, Luca Tagliaferri, MD, PhD²

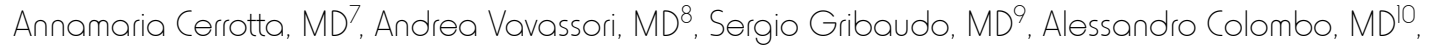
Francesco Lucà, MD", Raffaele Barbara, MD'2, Monica Mangoni, MD, PhD ${ }^{13}$, Francesco Marampon, MD, PhD!, Daniela Musio, MD!, Filippo Bellati, MD, PhD ${ }^{14}$, Ilary Ruscito, MD'4, Francesco Torcia, MD'14, Vincenzo Tombolini, MD!, Mattia Falchetto Osti, MD ${ }^{15}$, Vitaliana De Sanctis, MD ${ }^{15}$

'Department of Radiotherapy, Policlinico Umberto I, "Sapienza" University of Rome, Rome, Italy, 2Fondazione Policlinico Universitario A. Gemelli IRCCS, Dipartimento di Diagnostica per immagini, Radioterapia Oncologica ed Ematologia - Gemelli ART (Advanced Radiation Therapy), Interventional Oncology Center (IOC), Roma, Italy, ${ }^{3}$ Radiotherapy Unit, Azienda Ospedaliero Universitaria Ospedali Riuniti, Ancona, Italy, ${ }^{4}$ Radiotherapy Unit, S. Spirito Hospital, Pescara, Italy, ${ }^{5}$ Radiotherapy Unit, Department of Oncology, San Luca Hospital, Lucca, Italy, ${ }^{6}$ Radiotherapy Unit, Presidio Ospedaliero San Filippo Neri, Roma, Italy, ${ }^{7}$ Radiotherapy Unit, Fondazione IRCCS Istituto Nazionale dei Tumori, Milan, Italy, ${ }^{8}$ Division of Radiotherapy, IEO European Institute of Oncology. IRCCS, Milan, Italy, ${ }^{9}$ Department of Oncology Radiotherapy, A.O.U. Città della Salute e della Scienza di Torino, Turin, Italy, ${ }^{10}$ Division of Radiation Oncology, ASST-Lecco, Ospedale A. Manzoni, Lecco, Italy, "Presidente Fondazione Area Radiologica, Roma, Italy, ${ }^{2}$ UOC Radioterapia Oncologica, Azienda Ospedaliera "Brotzu", Cagliari, Italy, '3Radiotherapy Unit, Dipartimento di Scienze Biomediche, Sperimentali e Cliniche Mario Serio, University of Florence, Firenze, Italy, ${ }^{14}$ Department of Medicine and Surgery and Translational Medicine, Gynecology Oncology, Sant' Andrea Hospital, "Sapienza" University, Rome, Italy, ${ }^{15}$ Department of Medicine and Surgery and Translational Medicine, Radiotherapy Oncology, Sant' Andrea Hospital, "Sapienza" University, Rome, Italy
\end{abstract}

\begin{abstract}
Purpose: This systematic review focused on rare histological types of corpus uteri malignancy, including uterine carcinosarcoma (UCS), uterine clear cell carcinoma (UCCC), and uterine papillary serous carcinoma (UPSC), and it is proposed to assist with clinical decision-making. Adjuvant treatment decisions must be made based on available evidences. We mainly investigated the role of vaginal interventional radiotherapy (VIRt) in UCS, UCCC, and UPSC managements.

Material and methods: A systematic research using PubMed and Cochrane library was conducted to identify full articles evaluating the efficacy of VIRt in early-stage UPSC, UCCC, and UCS. A search in ClinicalTrials.gov was performed in order to detect ongoing or recently completed trials as well as in PROSPERO for ongoing or recently completed systematic reviews. Survival outcomes and toxicity rates were obtained.

Results: All studies were retrospective. For UCS, the number of evaluated patients was 432 . The 2- to 5-year average local control (LC) was 91\% (range, 74.2-96\%), disease-free survival (DFS) 88\% (range, 82-94\%), overall survival (OS) $79 \%$ (range, 53.8-84.3\%), the average 5-year cancer-specific survival (CSS) was 70\% (range, 70-94\%), and G3-G4 toxicity was $0 \%$. For UCCC, the number of investigated patients was 335 (UCCC - 124, mixed - 211), with an average 5-year LC of $100 \%$, DFS of $83 \%$ (range, $82-90 \%$ ), OS of $93 \%$ (range, $83-100 \%$ ), and G3-G4 toxicity of $0 \%$. For UPSC, the number of examined patients was 1,092 (UPSC - 866, mixed - 226). The average 5-year LC was 97\% (range, 87.1-100\%), DFS 84\% (range, 74.7-95.6\%), OS 93\% (range, 71.9-100\%), CSS 89\% (range, 78.9-94\%), and G3-G4 toxicity was 0\%.

Conclusions: These data suggest that in adequately selected early-stage UPSC and UCCC patients, VIRt alone may be suitable in women who underwent surgical staging and received adjuvant chemotherapy. In early-stage UCS, a multidisciplinary therapeutic approach has to be planned, considering high-rate of pelvic and distant relapses.

Key words: endometrial cancer, rare tumor, adjuvant treatment, brachytherapy, radiotherapy.

\footnotetext{
Address for correspondence: Sara Costantini, Radiotherapy Unit, Azienda Ospedaliero Universitaria Ospedali Riuniti, via Conca 71 60126, Ancona, Italy, phone: +39-071-596-4842, fax: +39-071-596-4838, e-mail: sara.costantini@ospedaliriuniti.marche.it
}

Received: 18.10 .2020 Accepted: 08.02 .2021 Published: 14.04 .2021 


\section{Purpose}

Worldwide, cancer of corpus uteri is the sixth most commonly diagnosed malignancy in women (4.4\% of the total cases of cancer) [1]. Uterine carcinosarcoma (UCS), uterine clear cell carcinoma (UCCC), and uterine papillary serous carcinoma (UPSC) are uncommon histological sub-types, and constitute less than $15 \%$ of all endometrial cancers [2-5]. These histologies represent an aggressive disease, characterized by local relapse and rapid metastatic dissemination, leading to a poor prognosis [2-5]. The distribution is predominant in older non-obese women and primarily affects African-American women [2-5].

Due to their rarity, prospective randomized studies have been hampered and thus, there is a lack of robust recommendations to support the optimal adjuvant treatment strategy in such patients. In this scenario, we conducted a systematic review to define the role of adjuvant vaginal interventional radiotherapy (VIRt) in UCS, UCCC, and UPSC managements. Also, the aim of this review was to propose it as a useful clinical tool for further expert consensus.

\section{Material and methods}

A systematic research using PubMed and Cochrane library was performed to identify relevant articles evaluating the efficacy of VIRt in patients with early-stage UCS, UCCC, and UPSC, published from January 1990 to July 2020. A search in ClinicalTrials.gov and PROSPERO was conducted to detect ongoing and recently completed trials and systematic reviews, respectively. The following medical subject headings (MeSH) and keywords were used: "endometrial neoplasms", "brachytherapy", "endovaginal radiotherapy", "uterine clear cell carcinoma", "uterine carcinosarcoma", and "uterine papillary serous carcinoma". The search was restricted to English language publications, including humans. Studies were eligible if patients had a proven early-stage UCS, UCCC, and UPSC treated with adjuvant VIRt. Review articles, conference papers, case reports, commentaries, letters, and book chapters were not included. Reference lists of previously published reviews were explored. Four independent investigators with $>5$ years of experience on endometrial cancer screened citations in titles and abstracts in order to identify appropriate papers. Eligible citations were retrieved for full-text reviews. Any disagreement was resolved by a consensus. When two articles appeared to report results with overlapping data, only the data representing the most recent publication were included in the review. The following parameters were obtained from all included studies: first author's surname, publication year, sample size, median age, treatment, and clinical outcomes, such as disease-free survival (DFS), local control (LC), overall survival (OS), cancer-specific survival (CSS), and toxicity rates.

\section{Results}

We organized the results by a histological sub-type. The following key areas were covered: 1) uterine carcino- sarcoma (UCS), 2) uterine clear cell carcinoma (UCCC), 3) uterine papillary serous carcinoma (UPSC). In total, there were no prospective studies. Based on selection criteria, only data from the VIRt treatment arms were extracted and considered for the analysis.

\section{Uterine carcinosarcoma (UCS)}

Literature search resulted in 511 articles. After exclusion by title and abstract, and after exclusion of conference papers, case reports, commentaries, letters, book chapters, reviews, and non-English language publications, 30 papers were assessed via full text for eligibility. Of these, 26 articles were excluded due to insufficient data, leaving 4 studies assessing the clinical efficacy of VIRt in DFS (Figure 1). All studies were retrospective [6-9]. Our review identified 432 patients with an average age of 67 years (range, 27-96 years) and a median follow-up of 35 months (range, 24-48 months). All patients underwent total abdominal hysterectomy and bilateral salpingooophorectomy (TAH/BSO). Pelvic lymph nodes dissection was performed in $79 \%$ to $97 \%$ cases, while para-aortic lymphadenectomy in $53 \%$ to $68 \%$ cases. Peritoneal cytology was performed in most patients (94-98\%), and omental sampling in $42 \%$ to $78 \%$ cases. The presence of positive lymphovascular invasion was reported in 191 patients out of 412 patients analyzed. Adjuvant chemotherapy was administered in $55 \%$ to $74 \%$ of patients, with intervals of at least one week between chemotherapy and VIRt. Chemotherapy consisted of platinum/taxane doublets. Adjuvant exclusive VIRt was delivered to the proximal two-thirds of the vagina and prescribed at an average dose of 21 Gy (range, 21-30 Gy) in 3-5 fractions, at a depth of $0.5 \mathrm{~cm}$ or at vaginal surface of the upper half of vagina. Studies including VIRt plus chemotherapy as a treatment, reported a vaginal cuff and pelvic relapse rate that ranged from $0 \%$ to $9 \%$ and from $12 \%$ to $45 \%$, respectively. The 2- to 5 -year average LC was 91\% (range, $74.2-96 \%$ ), DFS $88 \%$ (range, $82-94 \%$ ), and OS 79\% (range, $53.8-84.3 \%$ ). The average 5-year CSS was 70\% (range, $70-94 \%$ ) and G3-G4 toxicity was $0 \%$ (Table 1). In case of adjuvant treatment based on external beam radiotherapy plus VIRt and chemotherapy, both vaginal and pelvic relapse rate were $0-1 \%$. Moreover, the distant relapse rate ranged from $6 \%$ to $87 \%$ of patients (Table 2). Stage IB-II were associated with poor outcomes, including presence of lymphovascular invasion, age $>65$ years, and cervical involvement. Concurrent chemo-radiotherapy and VIRt (alone or boost) were associated with higher DSS and freedom from vaginal recurrence, respectively $[6,8,9]$. UCS showed worse outcomes when compared to UPSC, but these differences were not statistically significant in patients treated with chemotherapy plus VIRt [6, 7].

\section{Uterine clear cell carcinoma (UCCC)}

The literature search resulted in 507 articles. After the initial screening of titles and abstracts, 24 papers were identified. Of these, 18 articles were excluded due to insufficient data, leaving 6 studies assessing the clinical efficacy of VIRt in DFS (Figure 2). All studies were 

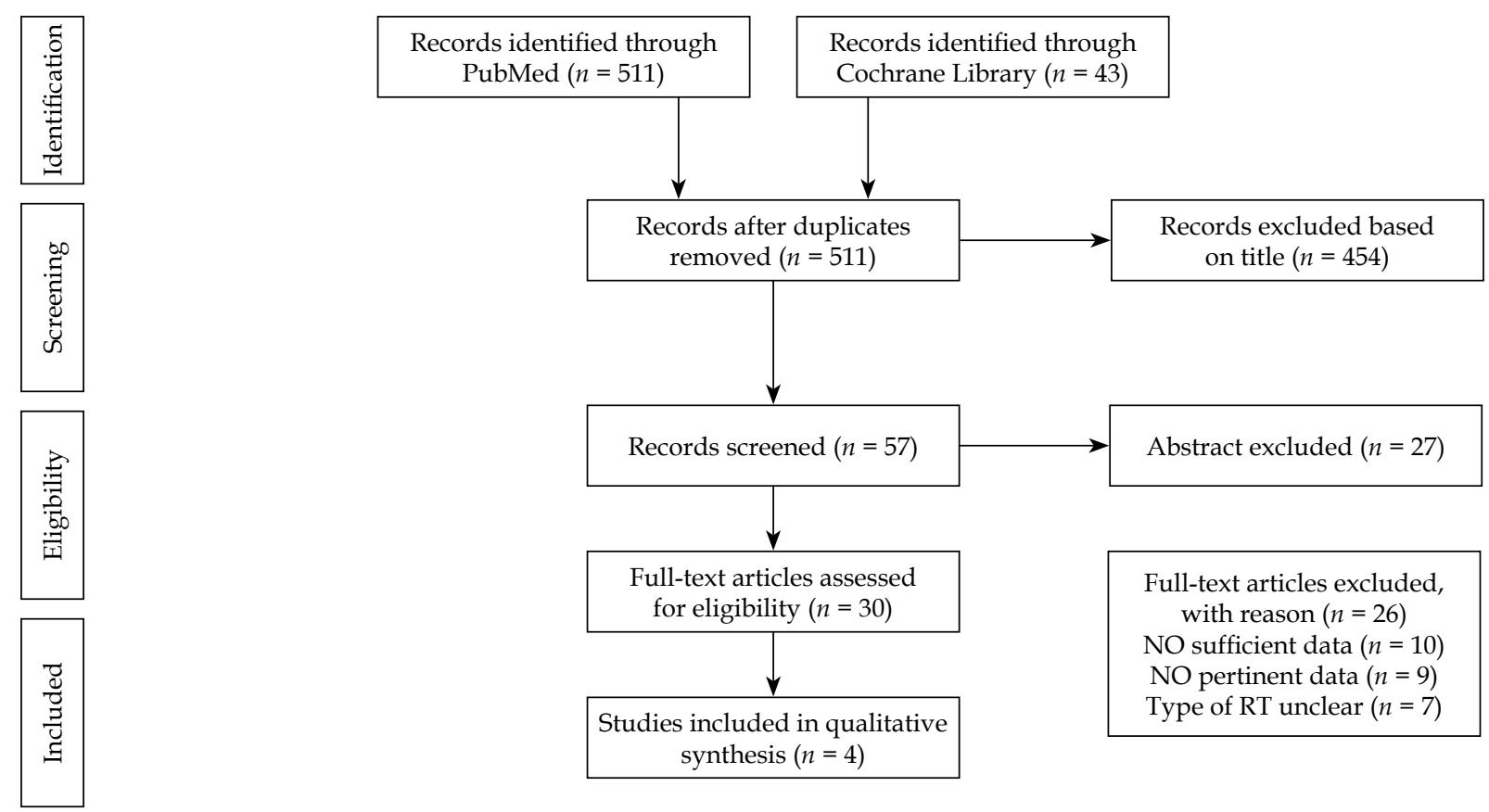

Fig. 1. PRISMA flow-chart for uterine carcinosarcoma (UCS)

retrospective [10-15]. In total, 335 patients (UCCC - 124, mixed - 211), with an average age of 65 years (range, 62-67 years) and a median follow-up of 35 months (range, 24-61 months) were enrolled. All but one paper [15] reported TAH/BSO, pelvic and para-aortic lymph nodes dissection, peritoneal cytology, omental sampling, and the presence of positive lymphovascular invasion. In those studies including not unique and specific histology, data concerning UCCC, UPSC, and mixed histologies were not separately considered. In 112 patients with mixed or pure UCCC endometrial carcinoma, pelvic and para-aortic lymph nodes dissection was reported in $81 \%$ and $62 \%$, respectively [15]. Adjuvant chemotherapy was administered in $34 \%$ to $100 \%$ of patients, with intervals of at least one week between chemotherapy and VIRt. Chemotherapy consisted of platinum/taxane doublets. Adjuvant VIRt was prescribed typically to the proximal twothirds of the vagina at an average dose of $21 \mathrm{~Gy}$ (range, 21-31.5 Gy) in 3-6 fractions, at a depth of $0.5 \mathrm{~cm}$ or at vaginal surface of the upper half of vagina. When specifically reported for UCCC histology, the 2- to 5-year average LC was $100 \%$, DFS $83 \%$ (range, $82-90 \%$ ), and OS 93\% (range, $83-100 \%$ ), with G3-G4 toxicity of $0 \%$ (Table 2) $[12,13]$.

In the study by Armbruster et al., in 112 patients with early-stage UCCC, the median OS was longer in patients who received adjuvant treatment compared to those who did not receive adjuvant therapy, but this difference was not statistically significant [15]. On multivariate analysis, age $\geq 70$, positive lymphovascular invasion [15] stage IB-IC $[12,14]$, and UCCC vs. mixed histology were associated with poorer outcomes. Better outcomes were associated with adjuvant therapy, i.e. chemotherapy plus radiotherapy and/or VIRt [11, 15]. In selected UCCC and UPSC stage I patients (with pelvic and aortic lymph nodes staging, omental sampling, and peritoneal wash- ing), exclusive VIRt (with or without chemotherapy) can be an option, in particular in stage IA patients (without myometrial invasion) $[10,12,14]$.

\section{Uterine papillary serous carcinoma (UPSC)}

Literature search details of the VIRt role in UPSC were described in our previous systematic review [16]. Here, we presented a brief overview of the main data (Figure 3). A total of 12 retrospective studies were finally analyzed on the basis of their relevance to the scope of this review [6, 10-14, 17-22]. Our review identified 1,092 patients (UPSC - 866, mixed - 226), with an average age of 67 years (range, 27-96 years), 924 with FIGO stage I disease (when specifically described 608 patients with stage IA and 46 with stage IB), and 66 patients with FIGO stage IC-II, with a median follow-up of 36 months (range, 21-62 months). All patients received TAH/BSO. Pelvic lymph nodes dissection was performed in most cases (79-100\%), and para-aortic lymphadenectomy was reported in $23 \%$ to $100 \%$ of cases. Peritoneal cytology ranged from $79 \%$ to $100 \%$, and omental sampling ranged from $38 \%$ to $100 \%$. The presence of positive lymphovascular invasion was reported in a median of $26 \%$ of patients (range, 4.7-70\%). Adjuvant chemotherapy was administered in $34 \%$ to $100 \%$ of patients, with intervals of at least one week between chemotherapy and VIRt, mainly consisted of platinum/taxane doublets. Adjuvant VIRt consisted of an average dose of 21 Gy (range, 12-37.5 Gy) in 3 fractions prescribed at a depth of $0.5-0.7 \mathrm{~cm}$ or at vaginal surface. Details of clinical outcomes are listed in Tables 1 and 2.

In patients treated with VIRt plus chemotherapy, the studies reported a median rate of vaginal cuff, pelvic, and distance relapse of $3 \%, 4 \%$, and $8 \%$, respectively.

The average 5-year LC was 97\% (range, 87.1-100\%), DFS 84\% (range, 74.7-95.6\%), OS 93\% (range, 71.9-100\%), 


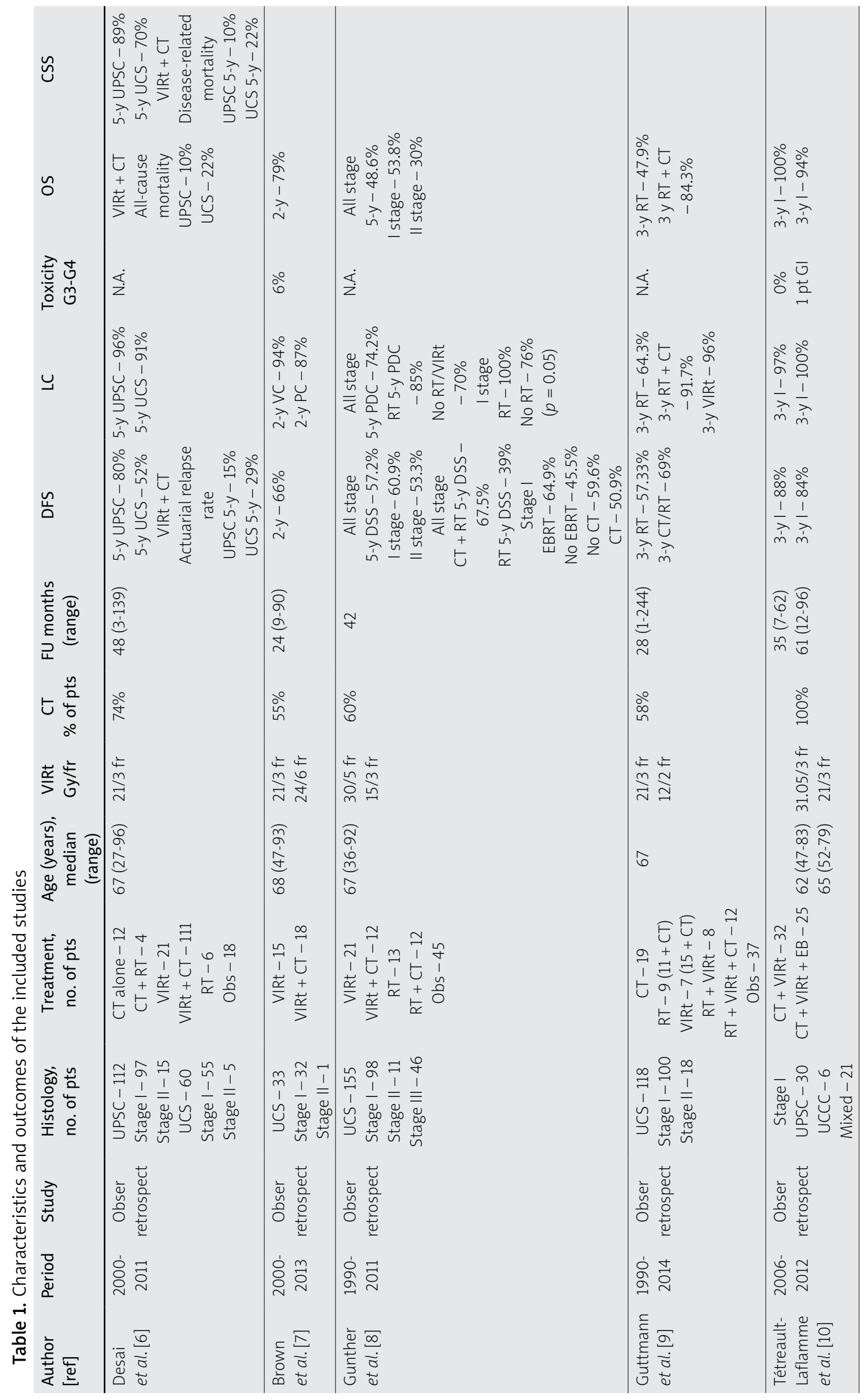




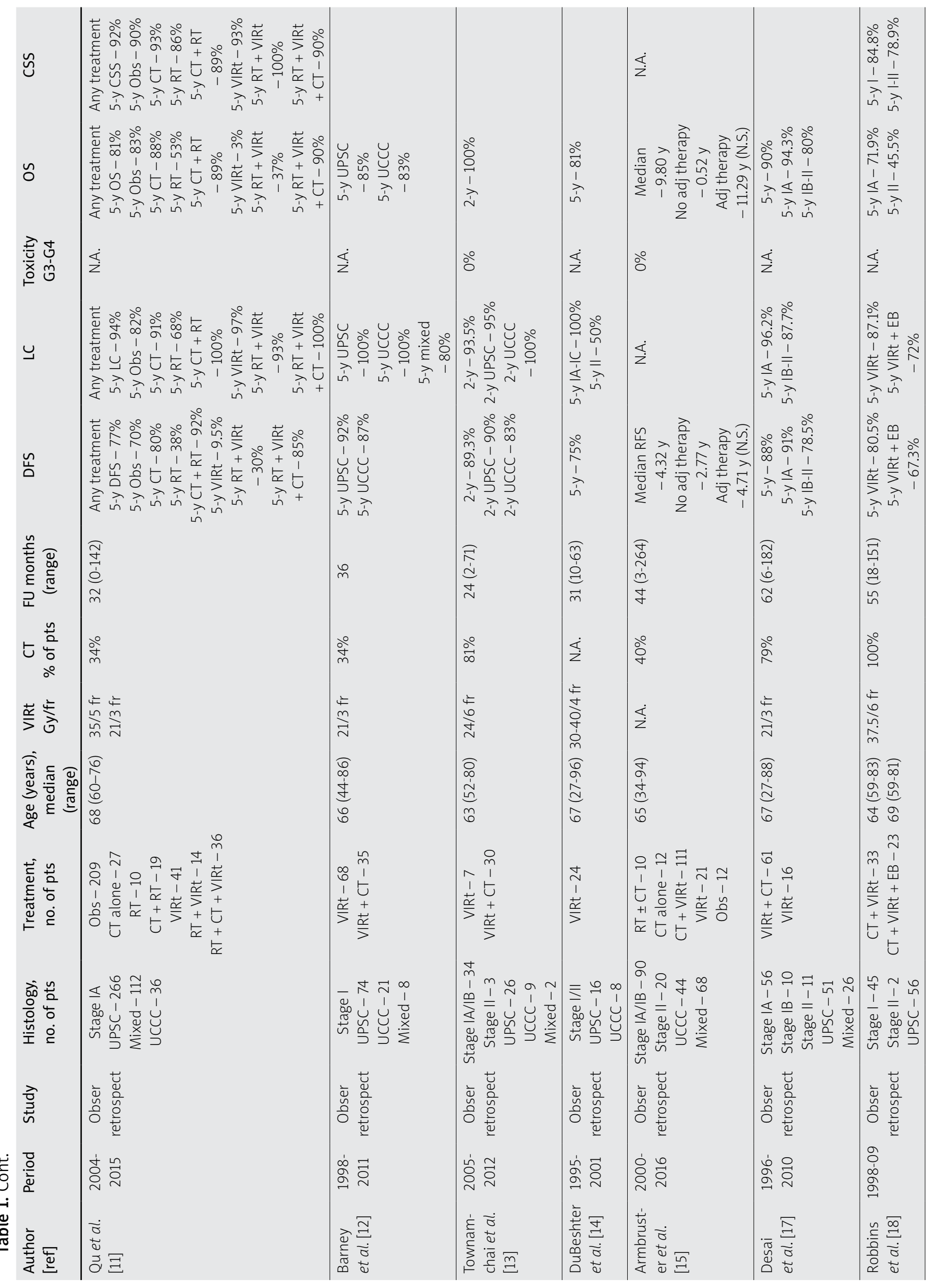




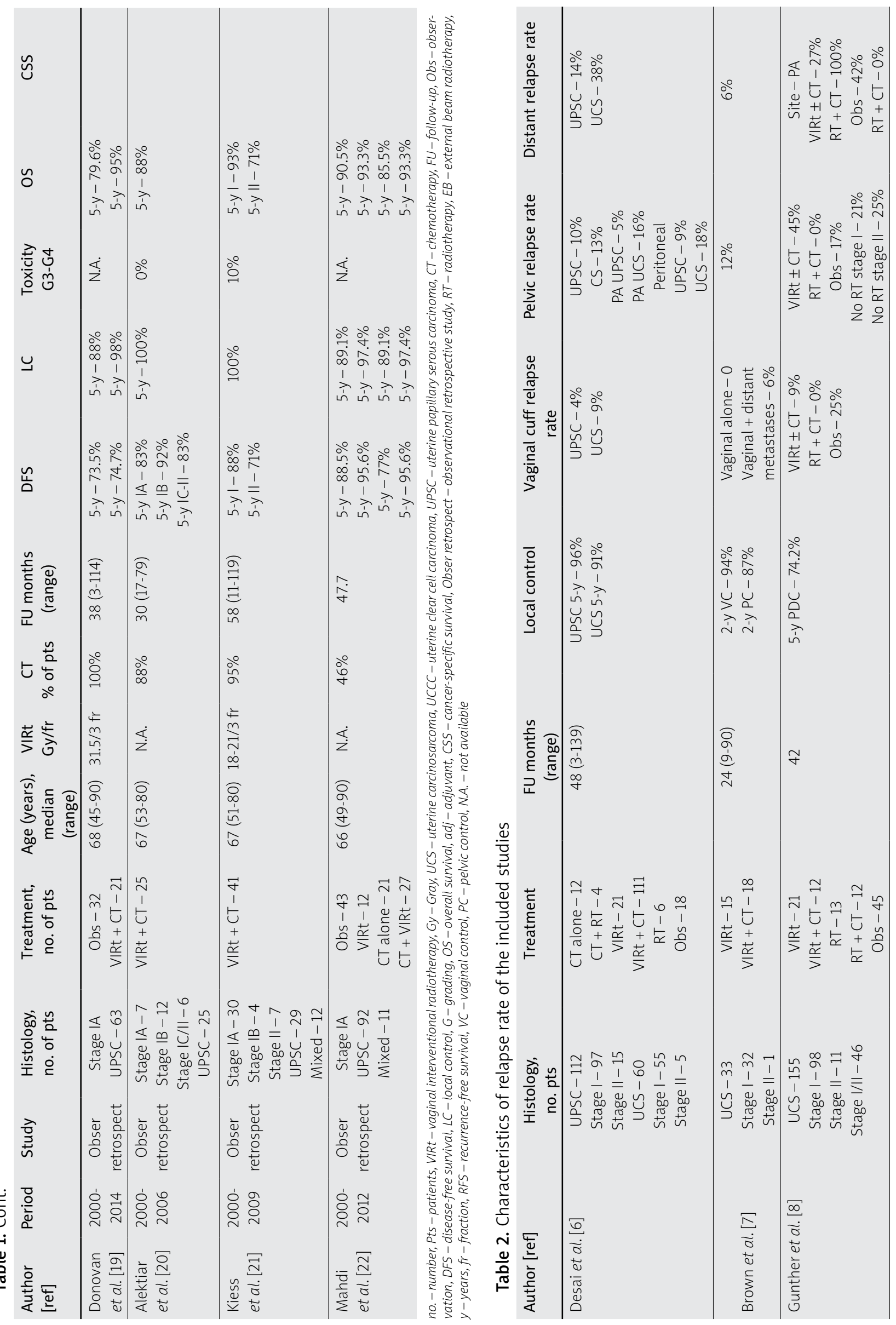




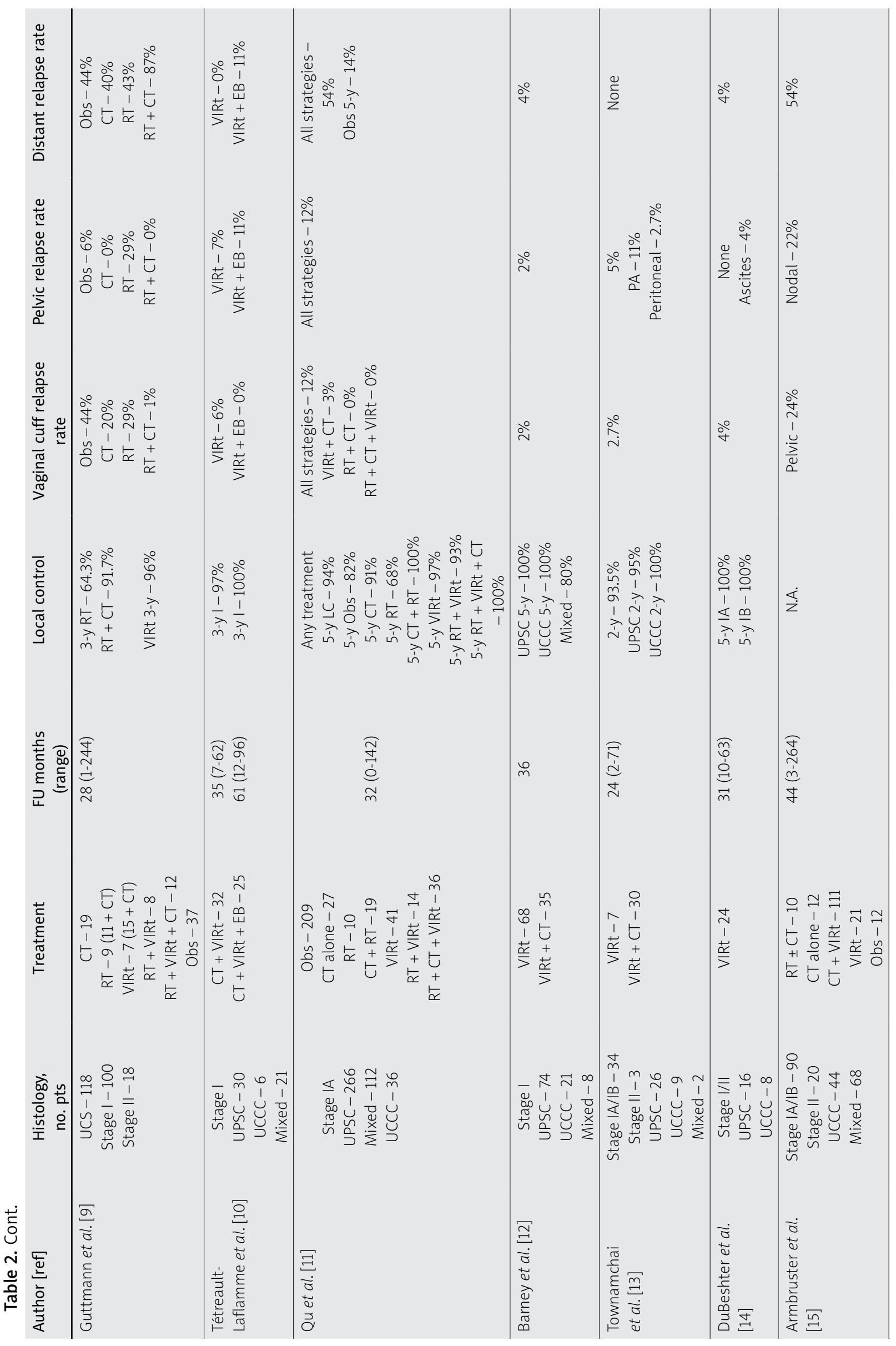




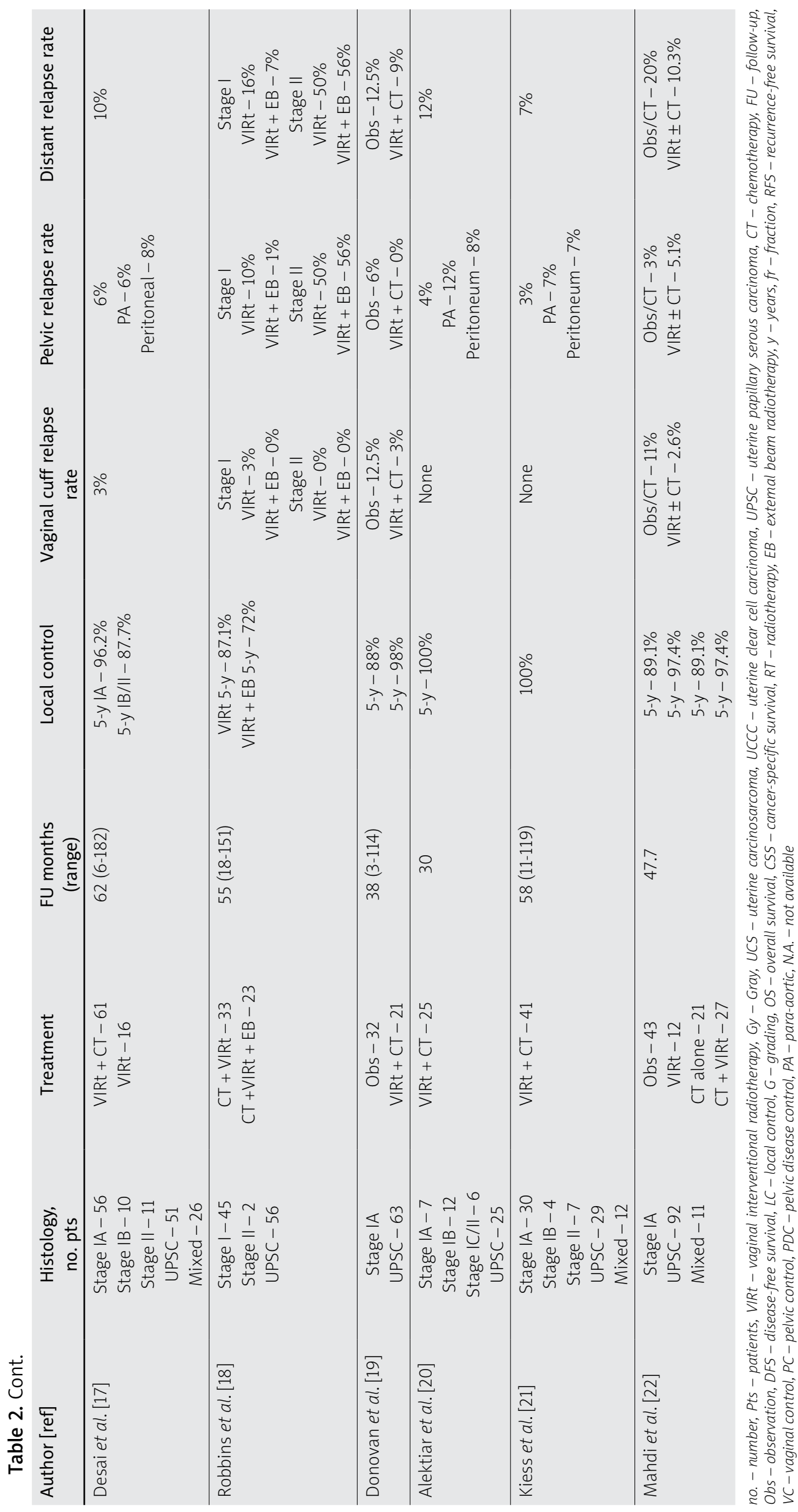




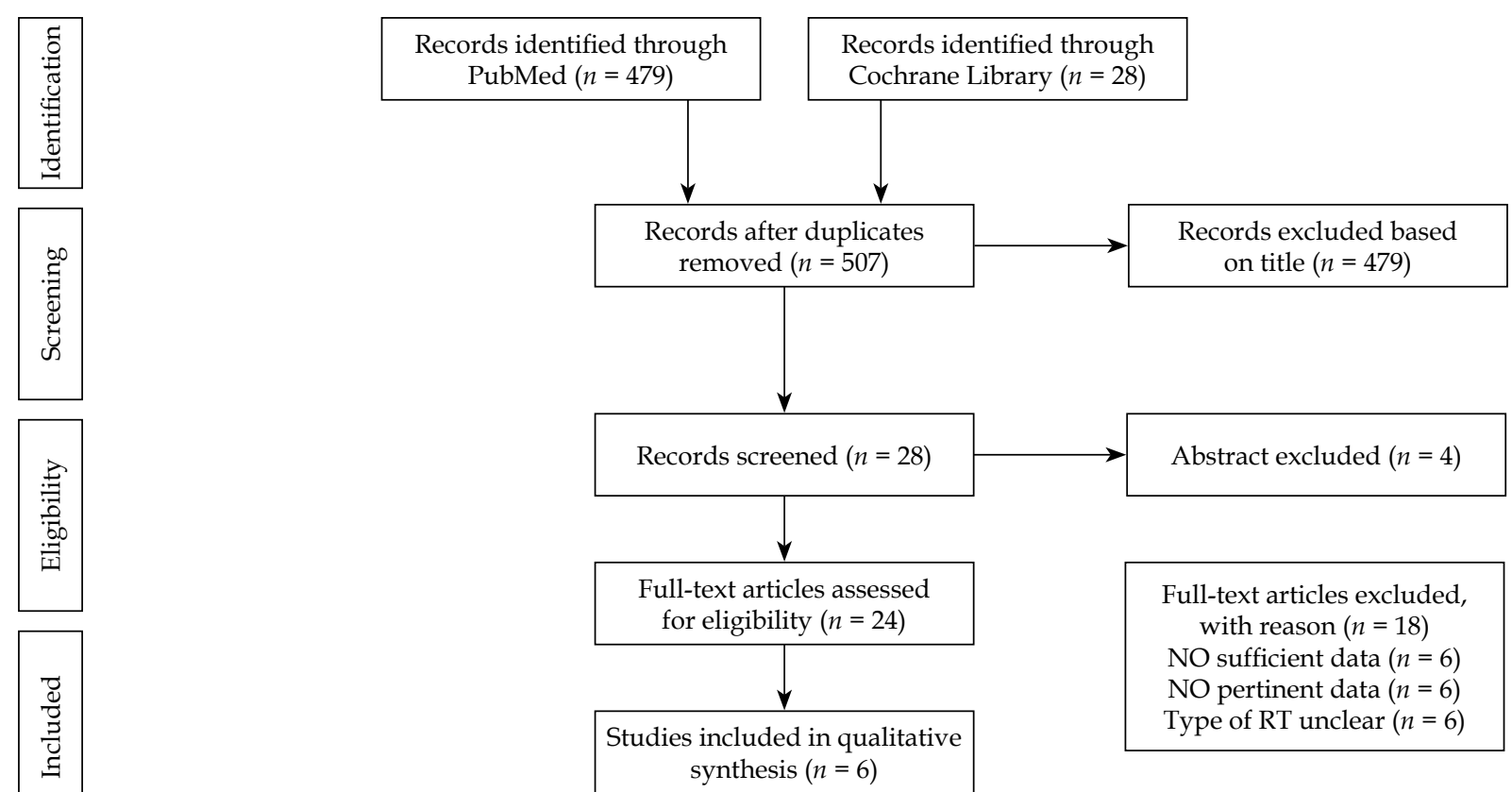

Fig. 2. PRISMA flow-chart for uterine clear cell carcinoma (UCCC)
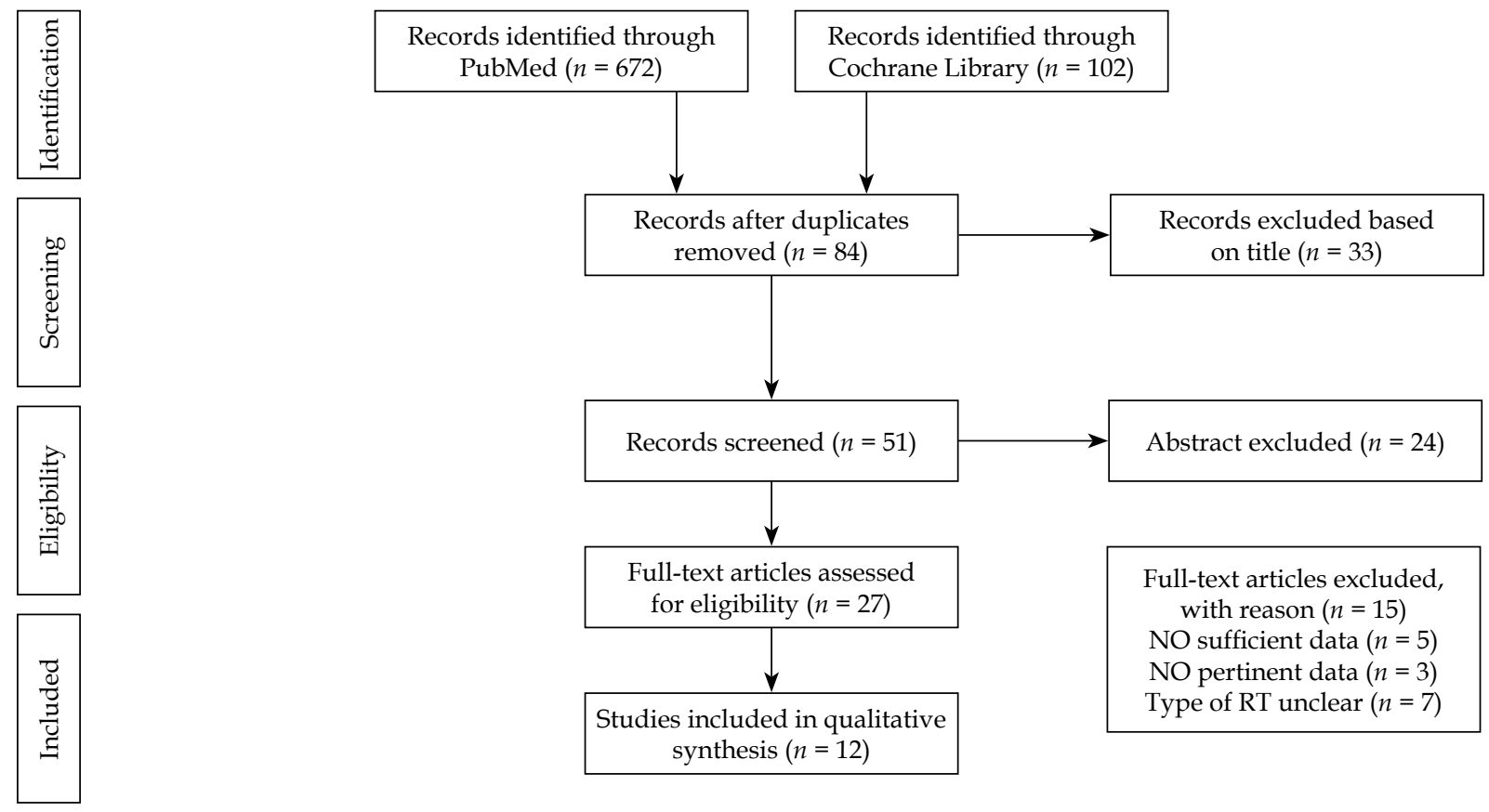

Fig. 3. PRISMA flow-chart for uterine papillary serous carcinoma (UPSC)

and CSS 89\% (range, 78.9-94\%). There was no evidence of severe toxicity. In a large series of 404 stage IA patients (UPSC - 266, UCCC - 26, mixed - 112) better outcomes (5-year DFS 85\%, LC 100\%, OS 90\%) were associated with chemotherapy plus radiotherapy and VIRt [11]. On the contrary, 2 studies showed similar results when chemotherapy plus VIRt was compared to chemotherapy plus radiotherapy and VIRt. The authors concluded that VIRt alone seemed to be as effective as radiotherapy in early-stage UPSC treated with surgery and adjuvant chemo- therapy, because no significant clinical benefit of adding pelvic radiotherapy was recorded $[10,18]$.

Four studies reported a comparison between VIRt plus chemotherapy and exclusive VIRt, and all of them showed very low vaginal recurrence rate with 2-5-year LC ranged between $93 \%$ to $100 \%$ and $2-5$-year OS from $83 \%$ to $100 \%$, with not statistical impact of chemotherapy in surgically staged patients $[12,13,17,22]$. Another study confirmed a local control in $96 \%$ of cases with exclusive adjuvant VIRt, and a risk of recurrence (13\%) increased 
according to the stage [14]. Finally, three studies showed a median rate of vaginal cuff, pelvic, and distance relapse of $0 \%, 4 \%$, and $9 \%$ in 117 UPSC and 12 mixed early-stage patients treated with VIRt and chemotherapy [19-21].

In these studies, when multivariate analysis was performed, myometrial invasion, lymphovascular invasion, and stage IB-IC/II were risk factors for poor DFS and/or OS, while chemotherapy had no impact on outcomes; however, in another large series of patients, both adjuvant VIRt and chemotherapy were associated with better local control and DFS [11-14, 17, 22]. Lymphadenectomy was associated with longer progression-free survival (PFS) [22].

\section{Discussion}

Radical surgery with total hysterectomy and bilateral salpingo-oophorectomy is the current standard treatment for non-metastatic UCS, UCCC, and UPSC [5, 23, 24]. The role of complete staging with systemic lymphadenectomy and omentectomy should be considered in early-stage diseases due to its improvement in survival and a decrease of overall recurrence rate, especially in UPSC [25-28].

Owing to the rarity of these tumors, there is no established consensus regarding adjuvant therapy. All suggestions for post-operative treatment are based on retrospective studies, limited by small simple size and/or heterogeneity of patients' characteristics, especially due to the infrequent subset of these histologies.

\section{Uterine carcinosarcoma (UCS)}

Chemotherapy seems to improve OS and DFS in patients affected by advanced-stage UCS, but its role in early-stage is unclear [29-32]. A recent study evaluated the role of chemotherapy, radiotherapy, and a combination of both after surgery in 3,447 women with stage I UCS. The 5-year OS for no treatment was $51.9 \%$ compared to $67.6 \%$ for chemotherapy, $57.9 \%$ for radiotherapy, and $73.9 \%$ for chemotherapy plus radiotherapy. Adjuvant chemotherapy (95\% CI: $0.52-0.79 \%, p<0.001$ ) and chemotherapy plus radiotherapy (95\% CI: $0.42-0.64 \%$, $p<0.001)$ were predictors for improved survival [33]. The efficacy of adjuvant radiotherapy in terms of LC and OS in patients with UCS has been reported in several retrospective studies [34-36]. However, the same results were not found in two prospective clinical trials [37, 38]. The phase III trial conducted by Gynecologic Oncology Group (GOG-150) enrolled 232 patients with stages I-IV primary UCS of the uterus or cervix. They were randomized to receive whole abdominal irradiation or chemotherapy (cisplatin/ifosfamide/mesna). The authors did not find a significant difference in relapse rate or OS between the two regimens, maybe due to relatively small sample size. This study has some limitations: whole abdominal radiation therapy is now rarely used, and the technique and dose prescribed are now considered obsolete. Moreover, carboplatin/paclitaxel is now the most commonly chosen first-line adjuvant chemotherapy regimen for UCS [37].
The European Organization for Research and Treatment of Cancer (EORTC) conducted a phase III trial with patients affected by stage I-II uterine sarcoma (including leiomyosarcoma, carcinosarcoma, and endometrial stromal sarcoma). Women were randomized to receive no therapy or pelvic radiotherapy as adjuvant treatment. No statistically significant differences were found in median PFS and OS between the two arms. However, patients affected by UCS and treated with radiotherapy had a better LC compared to those who did not receive adjuvant treatment. It should be noted that these histological subtypes, which used to be grouped as uterine sarcomas, are no longer grouped together [38].

In a series of 118 UCS early-stage patients, an advantage in LC has been demonstrated in patients treated with combined radiation therapy (external beam plus VIRt) and chemotherapy [9]. In contrast, in a study by Gunther et al., external beam radiotherapy was associated with better loco-regional control and time to distant metastatic spread without significant impact to OS compared to VIRt alone [8].

In a retrospective study of patients affected by stage I-II UCS, external beam radiotherapy and VIRt were compared in terms of effectiveness. VIRt alone did not have worse OS and DFS compared to external beam radiotherapy. The authors suggested VIRt as an alternative to pelvic radiotherapy in patients adequately surgically staged and undergone adjuvant chemotherapy [39]. Moreover, in SEER retrospective analysis, the use of VIRt alone showed a substantial increase over time, from $4.5 \%$ during 19881999 to $12.5 \%$ during 2005-2010. In other studies, the "cuff and chemo" approach had the largest survival benefit in women with node-negative UCS [6, 40].

In conclusion, currently, there is a lack of consensus on the optimal therapeutic strategy in early-stage UCS [6]. Moreover, there is no clinical trial that suggests a survival benefit with adjuvant radiotherapy (external beam and/or VIRt) with or without chemotherapy. Results of several recent studies provided evidence regarding the importance of adequate surgical staging and comparative effectiveness of external beam radiotherapy versus VIRt. Despite a worse acute toxicity profile, VIRt and chemotherapy seemed to be an effective alternative strategy to external beam radiotherapy in UCS early-stage patients [39-41].

\section{Uterine clear cell (UCCC) and uterine papillary serous carcinomas (UPSC)}

A multi-institutional study published in 2009 demonstrated the role of systemic therapy for early-stage UPSC. PFS and CSS for chemotherapy-treated patients were more favorable than for those who did not receive chemotherapy. Five-year PFS and CSS rates were $81.5 \%$ and $87.6 \%$ in chemo-radiotherapy group, $64.1 \%$ and $59.5 \%$ in radiotherapy alone group, and $64.7 \%$ and $70.2 \%$ in observational group, respectively [42]. Platinum-based chemotherapy improved the DFS and OS rates in patients with stage I UPSC, and vaginal cuff radiation provided LC [43]. PORTEC-3 trial investigated the role of chemotherapy plus radiotherapy versus radiotherapy alone in pa- 
tients affected by high-risk endometrial cancer. The combined treatment significantly improved the 5-year failure-free survival (FFS) compared to radiotherapy alone (95\% CI: $75.5 \%$ vs. $68.6 \%, p=0.067$ ). No significant difference in OS was observed (95\% CI: $81.8 \%$ vs. $76.7 \%$ $p=0.213$ ). Patients with UPSC had much benefit from combined treatment in terms of FFS compared to the other histologies ( $\mathrm{HR}=0.44,95 \% \mathrm{CI}: 0.31-0.60 \%$ ). However, this benefit was not statistically significant because of the small number of UPSC cases [44].

Contrary to previous reported data, international guidelines and studies suggested exclusive radiotherapy as adjuvant treatment for patients with non-endometrioid uterine carcinoma, and VIRt may be considered an option in early-stage UPSC and UCCC $[12,13,16,17,45,46]$. A national large-scale study of cancer-specific outcomes demonstrated that VIRt was the only treatment to significantly improve cancer-specific outcomes in stages I-II cohort, and that both VIRt and chemotherapy appeared beneficial in early-stage patients with papillary-serous histology [47]

Qu et al. observed a significant advantage from VIRt as adjuvant treatment associated with chemotherapy in improving local control, even if failed in detection of a dose-response relationship between radiation dose and local control [11]. The first study available in literature comparing external beam radiotherapy and VIRt in 87 women with stage I UPSC or UCCC, described the same effectiveness of VIRt alone as external beam radiotherapy and VIRt when associated with surgery and adjuvant chemotherapy [10].

In their multi-institutional retrospective review, Madhi et al. confirmed a lower risk of vaginal recurrence in patients with early non-invasive UPSC, who underwent VIRt \pm chemotherapy compared to patients who received chemotherapy alone or no treatment $(2.6 \%$ vs. $10.9 \%)$. In contrast, they did not detect an improvement in PFS or OS [22]. These results were confirmed in other retrospective studies $[17,20,21]$.

A retrospective analysis of adjuvant therapy for patients affected by stage I-II UPSC showed an increasing use of VIRt and chemotherapy, and a decrease of external beam radiotherapy from 1998 to 2012. In these patients, VIRt and chemotherapy were associated with a reduction of mortality not seen with the use of external beam radiotherapy [48]. Donovan et al. advocates the use of chemotherapy and VIRt for patients with stage IA UPSC, given the low-rate of pelvic recurrence [19].

Finally, a recent phase III trial showed no different outcomes in terms of recurrence-free survival (RFS) and OS, in patients affected by high-risk endometrial cancer randomized to receive external beam radiotherapy vs. chemotherapy plus VIRt. The 60-month RFS was 0.76 (95\% CI: 0.70$0.81 \%)$ for radiotherapy and 0.76 (95\% CI: $0.70-0.81 \%)$ for chemotherapy plus VIRt (HR $=0.92,90 \%$ CI: $0.69-1.23 \%)$. The 60 -month OS was 0.87 (95\% CI: $0.83-0.91 \%$ ) for radiotherapy and 0.85 (95\% CI: $0.81-0.90 \%)$ for chemotherapy plus VIRt (HR $=1.04,90 \%$ CI: $0.71-1.52 \%$ ) [49].

There is no consensus regarding the optimal dosefractionation schedule for the delivery of VIRt. The 2014
American Brachytherapy Society (ABS) update survey reported that $7 \mathrm{~Gy}$ for three fractions is the most common schedule for post-operative VIRt alone. Nevertheless, a wide variation in dose schedules was noted [50]. In this review, the median total surface dose was $21 \mathrm{~Gy}$ in 3 fractions (range, 15-37.5 Gy in 3-6 fractions).

Several studies confirmed a lower if any acute gastrointestinal and genitourinary toxicity in the VIRt treatment with or without chemotherapy [7, 10, 13, 15, 20, 21], resulting in a good quality of life as well as PORTEC-2 trial [48-52]. To note, PORTEC-2 was a randomized trial for women with intermediate-risk endometrioid uterine cancer, comparing pelvic external beam radiotherapy with VIRt in terms of LC and toxicity [53].

These data are consistent with a recent review, in which authors established that acute endovaginal toxicity may occur in less than $20.6 \%$ of cases, while late vaginal toxicity G1-G2 may appear in less than $27.7 \%$ and G3-G4 in less than 2\% [54]. Furthermore, limited available evidence suggested that there is no difference in terms of acute and late toxicity between low-dose-rate and highdose-rate VIRt [55], or using high-dose-rate VIRt short schedule [56].

Overall, these results are informative, but not completely generalizable. The major limitation of this review was the retrospective nature of the included studies. Based on the assumption that retrospective studies start with a "low quality" rating [57], the usefulness of adjuvant VIRt was difficult to interpret. Certainly, the surgical approach remains the gold standard in this setting of patients.

\section{Conclusions}

This systematic review suggests the potential role of VIRt in the management of non-endometrioid carcinoma of corpus uteri. Overall, adjuvant VIRt seems to be helpful to improve clinical outcomes, especially DFS and LC. No definitive conclusions can be drawn, mainly due to the lack of adequately powered randomized studies. At present, adjuvant VIRt may be justified, based on its positive effect on local control, while minimizing severe toxicity. Undeniably, the proper treatment strategy in patients with non-endometrioid carcinoma of corpus uteri should be described in a prospective trial.

\section{Disclosure}

The authors report no conflict of interest.

\section{References}

1. Bray F, Ferlay J, Soerjomataram I et al. Global cancer statistics 2018: GLOBOCAN estimates of incidence and mortality worldwide for 36 cancers in 185 countries. CA Cancer J Clin 2018; 68: 394-424.

2. Boruta DM 2nd, Gehrig PA, Fader AN et al. Management of women with uterine papillary serous cancer: a Society of Gynecologic Oncology (SGO) review. Gynecol Oncol 2009; 115: 142-153.

3. Olawaiye AB, Boruta DM 2nd. Management of women with clear cell endometrial cancer: a Society of Gynecologic Oncology (SGO) review. Gynecol Oncol 2009; 113: 277-283. 
4. Cantrell LA, Blank SV, Duska LR. Uterine carcinosarcoma: a review of the literature. Gynecol Oncol 2015; 137: 581-588.

5. Elshaikh MA, Modh A, Jhingran A. Executive summary of the American Radium Society Appropriate Use Criteria for management of uterine carcinosarcoma. Gynecol Oncol 2020; 158: 460-466.

6. Desai NB, Kollmeier MA, Makker V et al. Comparison of outcomes in early stage uterine carcinosarcoma and uterine serous carcinoma. Gynecol Oncol 2014; 135: 49-53.

7. Brown LC, Petersen IA, Haddock MG et al. Vaginal brachytherapy for early-stage carcinosarcoma of the uterus. Brachytherapy 2015; 14: 433-439.

8. Gunther JR, Christensen EN, Allen PK et al. Role of radiation therapy in the multidisciplinary management of uterine carcinosarcoma. Int J Gynecol Cancer 2018; 28: 114-121.

9. Guttmann DM, Li H, Sevak P et al. The impact of adjuvant therapy on survival and recurrence patterns in women with early-stage uterine carcinosarcoma: a multi-institutional study. Int J Gynecol Cancer 2016; 26: 141-148.

10. Tétreault-Laflamme A, Nguyen-Huynh TV, Carrier JF et al. Adjuvant chemotherapy and vaginal vault brachytherapy with or without pelvic radiotherapy for stage 1 papillary serous or clear cell endometrial cancer. Int J Gynecol Cancer 2016; 26: 301-306.

11. Qu M, Velker VM, Leung E et al. The role of adjuvant therapy in stage IA serous and clear cell uterine cancer: a multiinstitutional pooled analysis. Gynecol Oncol 2018; 149: 283-290.

12. Barney BM, Petersen IA, Mariani A et al. The role of vaginal brachytherapy in the treatment of surgical stage I papillary serous or clear cell endometrial cancer. Int J Radiat Oncol Biol Phys 2013; 85: 109-115.

13. Townamchai K, Berkowitz R, Bhagwat $M$ et al. Vaginal brachytherapy for early stage uterine papillary serous and clear cell endometrial cancer. Gynecol Oncol 2013; 129: 18-21.

14. DuBeshter B, Estler K, Altobelli K et al. High-dose rate brachytherapy for stage I/II papillary serous or clear cell endometrial cancer. Gynecol Oncol 2004; 94: 383-386.

15. Armbruster SD, Previs R, Soliman PT et al. Clinicopathologic features and treatment in patients with early stage uterine clear cell carcinoma: a 16-year experience. Gynecol Oncol 2019; 154: 328-332.

16. Lancellotta V, De Felice F, Vicenzi L et al. The role of vaginal brachytherapy in stage I endometrial serous cancer: a systematic review. J Contemp Brachytherapy 2020; 12: 61-66.

17. Desai NB, Kiess AP, Kollmeier MA et al. Patterns of relapse in stage I-II uterine papillary serous carcinoma treated with adjuvant intravaginal radiation (IVRT) with or without chemotherapy. Gynecol Oncol 2013; 131: 604-608.

18. Robbins JR, Siddiqui MS, Al-Wahab Z et al. Clinical outcomes of adjuvant chemotherapy and vaginal brachytherapy with or without pelvic radiation for surgical stage I-II uterine serous carcinoma. Eur J Gynaecol Oncol 2012; 33: 449-454.

19. Donovan E, Reade CJ, Eiriksson LR et al. Outcomes of adjuvant therapy for stage IA serous endometrial cancer. Cureus 2018; 10: e3387.

20. Alektiar KM, Makker V, Abu-Rustum NR et al. Concurrent carboplatin/paclitaxel and intravaginal radiation in surgical stage I-II serous endometrial cancer. Gynecol Oncol 2009; 112: 142-145.

21. Kiess AP, Damast S, Makker V et al. Five-year outcomes of adjuvant carboplatin/paclitaxel chemotherapy and intravaginal radiation for stage I-II papillary serous endometrial cancer. Gynecol Oncol 2012; 127: 321-325.

22. Mahdi H, Rose PG, Elshaikh MA et al. Adjuvant vaginal brachytherapy decreases the risk of vaginal recurrence in patients with stage I non-invasive uterine papillary serous carcinoma. A multi-institutional study. Gynecol Oncol 2015; 136: 529-533.
23. Patsavas K, Woessner J, Gielda B. Optimal surgical debulking in uterine papillary serous carcinoma affects survival. Gynecol Oncol 2011; 121: 581-585.

24. Hasegawa K, Nagao S, Yasuda M. Gynecologic Cancer InterGroup (GCIG) consensus review for clear cell carcinoma of the uterine corpus and cervix. Int J Gynecol Cancer 2014; 24: S90-S95.

25. Versluis MAC, Pielsticker C, van der Aa MA et al. Lymphadenectomy and adjuvant therapy improve survival with uterine carcinosarcoma: a large retrospective cohort study. Oncology 2018; 95: 100-108.

26. Mahdi H, Lockhart D, Moselmi-Kebria M. Prognostic impact of lymphadenectomy in uterine clear cell carcinoma. J Gynecol Oncol 2015; 26: 134-140.

27. Thomas MB, Mariani A, Cliby WA. Role of systematic lymphadenectomy and adjuvant therapy in stage I uterine papillary serous carcinoma. Gynecol Oncol 2007; 107: 186-189.

28. Kaban A, Topuz S, Erdem B et al. Is omentectomy necessary for non-endometrioid endometrial cancer. Gynecol Obstet Invest 2018; 83: 482-486.

29. Homesley HD, Filiaci V, Markman M et al. Phase III trial of ifosfamide with or without paclitaxel in advanced uterine carcinosarcoma: a Gynecologic Oncologic Group Study. J Clin Oncol 2007; 25: 526-531.

30. Powell MA, Filiaci VL, Rose PG. Phase II evaluation of paclitaxel and carboplatin in the treatment of carcinosarcoma of the uterus: a Gynecologic Oncology Group study. J Clin Oncol 2010; 28: 2727-2731.

31. Dandumadi RK, Aslam S, Walji N et al. Chemotherapy for uterine carcinosarcoma with carboplatin, ifosfamide and mesna. Anticancer Res 2015; 35: 4841-4848.

32. Sutton G, Kauderer J, Carson LF et al. Adjuvant ifosfamide and cisplatin in patients with completely resected stage I or II carcinosarcomas (mixed mesodermal tumors) of the uterus: a Gynecologic Oncology Group study. Gynecol Oncol 2005; 96: 630-634.

33. Cho S, Chan JK, Kapp D. Does chemotherapy or radiation benefit surgical stage I uterine carcinosarcoma patients? Am J Obstet Gynecol 2020; 222: 383-384.

34. Wright JD, Seshan VE, Shah M et al. The role of radiation in improving survival for early-stage carcinosarcoma and leiomyosarcoma. Am J Obstet Gynecol 2008; 199: 536.e1-8.

35. Smith DC, MacDonal OK, Gaffney DK. The impact of adjuvant radiation therapy on survival in women with uterine carcinosarcoma. Radiother Oncol 2008; 88: 227-232.

36. Lee T. Adjuvant pelvic radiotherapy for uterine carcinosarcoma in a high risk population. Eur J Surg Oncol 2001; 27: 282-285.

37. Wolfson AH, Brady MF, Rocereto T et al. A Gynecological Oncology Group phase III Trial of whole abdominal irradiation (WAI) vs Cisplatin-Ifosfamide-Mesna (cim) as post-surgical therapy in stage I-IV carcinosarcoma (CS) of the uterus. Gynecol Oncol 2007; 107: 177-185.

38. Reed NS, Mangioni C, Halmstrom $\mathrm{H}$ et al. Phase III randomized study to evaluate the role of adjuvant pelvic radiotherapy in the treatment of uterine sarcomas stages I-II: an European Organisation for Research and Treatment of Cancer Gynaecological Cancer Group Study (protocol 55874). Eur J Cancer 2008; 44: 808-818.

39. Patel N, Hegarty SE, Cantrell LA et al. Evaluation of brachytherapy and external beam radiation therapy for early stage, node-negative uterine carcinosarcoma. Brachytherapy 2015; 14: 606-612.

40. Seagle BL, Kanis M, Kocherginsky M et al. Stage I uterine carcinosarcoma: Matched cohort analyses for lymphadenectomy, chemotherapy, and brachytherapy. Gynecol Oncol 2017; 145: 71-77. 
41. Randall ME, Filiaci V, McMeekin DS et al. Phase III trial: adjuvant pelvic radiation therapy versus vaginal brachytherapy plus paclitaxel/carboplatin in high-intermediate and high-risk early-stage endometrial cancer. J Clin Oncol 2019; 37: 1810-1818.

42. Fader AN, Richard RD, O'Malley DM et al. Platinum/Taxane-based chemotherapy with or without radiation therapy favorable impacts survival outcomes in stage I uterine papillary serous carcinoma. Cancer 2009; 115: 2119-2127.

43. Kelly MG, O'Malley DM, Hui P et al. Improved survival in surgical stage I patients with uterine papillary serous carcinoma (UPSC) treated with adjuvant platinum-based chemotherapy. Gynecol Oncol 2005; 98: 353-359.

44. de Boer SM, Powell ME, Mileshkin L et al. Adjuvant chemoradiotherapy alone for women with high-risk endometrial cancer (PORTEC-3): final results of an international, open-label, multicentre, randomized, phase 3 trial. Lancet Oncol 2018; 19: 295-309.

45. National Comprehensive Cancer Network. NCCN Clinical Practice Guidelines in Oncology: uterine neoplasms. Available at: https://www.nccn.org/professionals/physician_ gls/pdf/uterine.pdf

46. Colombo N, Creutzberg C, Amant F et al. ESMO-ESGOESTRO consensus conference on endometrial cancer: Diagnosis, treatment and follow-up. Radiother Oncol 2015; 117: 559-581.

47. Xiang M, English DP, Kidd EA. National patterns of care and cancer-specific outcomes of adjuvant treatment in patients with serous and clear cell endometrial carcinoma. Gynecol Oncol 2019; 152: 599-604.

48. Cham S, Huang Y, Terga AI. Utility of radiation therapy for early-stage uterine papillary serous carcinoma. Gynecol Oncol 2017; 145: 269-276.

49. Randall ME, Filiaci V, McMeekin S et al. Phase III trial: adjuvant pelvic radiation therapy versus vaginal brachytherapy plus paclitaxel/carboplatin in high-intermediate and highrisk early stage endometrial cancer. J Clin Oncol 2019; 20: 1810-1818.

50. Harkenrider MM, Block AM, Alektiar KM et al. Adjuvant vaginal brachytherapy for early stage endometrial cancer: a comprehensive review. Brachytherapy 2017; 16: 95-108.

51. Nout RA, PutterH, Jürgenliemk-Schulz IM et al. Quality of life after pelvic radiotherapy or vaginal brachytherapy for endometrial cancer: first results of the randomized PORTEC-2 trial. J Clin Oncol 2009; 27: 3547-3556.

52. Nout RA, Putter H, Jürgenliemk-Schulz IM et al. Five-year quality of life of endometrial cancer patients treated in the randomised Post Operative Radiation Therapy in Endometrial Cancer (PORTEC-2) trial and comparison with norm data. Eur J Cancer 2012; 48: 1638-1648.

53. Nout RA, Smit VT, Putter H et al. Vaginal brachytherapy versus pelvic external beam radiotherapy for patients with endometrial cancer of high-intermediate risk (PORTEC-2): an open-label, non-inferiority, randomised trial. Lancet 2010; 375: 816-823.

54. Delishaj D, Barcellini A, D'Amico R et al. Vaginal toxicity after high-dose-rate endovaginal brachytherapy: 20 years of results. J Contemp Brachytherapy 2018; 10: 559-566.

55. Autorino R, Tagliaferri L, Campitelli M et al. EROS study: evaluation between high-dose-rate and low-dose-rate vaginal interventional radiotherapy (brachytherapy) in terms of overall survival and rate of stenosis. J Contemp Brachytherapy 2018; 10: 315-320.

56. De Sanctis V, Musio D, De Felice F. One-week vaginal brachytherapy schedule as exclusive adjuvant post-operative treatment in intermediate- and high-intermediate-risk endometrial cancer patients. J Contemp Brachytherapy 2020; 12: 124-130.

57. Guyatt GH, Oxman AD, Vist GE et al.; GRADE Working Group. GRADE: an emerging consensus on rating quality of evidence and strength of recommendations. BMJ 2008; 336: 924-926. 